\title{
REFERENCES
}

1. S. P. Frankel, Convergence rates of iterative treatments of partial differential equations, Math. Tables and Aids to Comp. 4, 65 (1950).

2. R. V. Southwell, Relaxation methods in physical sciences, Oxford Press, 1948.

3. J. L. Synge, A geometrical interpretation of the relaxation method, Q. Appl. Math. 2, 87 (1944).

4. H. D. Huskey, Characteristics of the INA computer, Math. Tables and Aids to Comp. 4, 103 (1950).

\section{THE STABILITY EQUATION WITH PERIODIC COEFFICIENTS*}

\section{By HIRSH COHEN (Haifa, Israel)}

In a large number of physical problems involving periodic motion, dynamic stability considerations result in stability differential equations which have periodic coefficients. In particular, if the physical system is described by a non-linear second order ordinary differential equation, a second order equation of the Floquet type appears. That this is not an isolated case becomes apparent if one reviews the large volume of non-linear mechanics literature of the past few years. The problem to be discussed in this note is even more specialized than the one just introduced but the same review through the literature will reveal that it is an important case. This is the stability problem which results when the non-linear element has small effect on the system, i.e., when the resultant motion is near to the motion of the linearized system.

As an example consider the van der Pol equation

$$
y^{\prime \prime}-\epsilon\left(1-y^{2}\right) y^{\prime}+y=0
$$

where primes refer to differentiation with respect to $t$. If $y$ is taken to be of 0 (1) then $\epsilon \ll 1$. The usual stability considerations involve the addition of a small (of order $\epsilon$ ) time-dependent function, $v(t)$, to an exact or approximate solution $y_{0}(t)$. On substitution into (1) of $y=y_{0}+v(t)$, the equation of first order in $v(t)$ is

$$
v^{\prime \prime}-\epsilon\left(1-y_{0}^{2}\right) v^{\prime}+\left(1+2 \epsilon y_{0} y_{0}^{\prime}\right) v=0 .
$$

If the solution is to be a periodic approximation to $y$, then $y_{0}$ is periodic and (2) represents an example of the general equation dealt with herein, namely

$$
u^{\prime \prime}+\epsilon p(t) u^{\prime}+\epsilon\left(q_{1}(t)+\frac{1}{\epsilon}\right) u=0,
$$

where $u$ is the disturbance function being used to "test" some system, and $p(t)$ and $q_{1}(t)$ are periodic functions of period $2 \pi / \omega$.

It can be seen immediately that the Mathieu equation is a special case of (3). Furthermore, it would appear useful to remove the first order term in (3) and thus reduce it to at worst a Hill equation. This may be done by the substitution

$$
u=v(t) \exp \left[-\frac{\epsilon}{2} \int p(t), d t\right] \text {. }
$$

*Received December 19, 1951. 
with the resulting equation in $v$

$$
v^{\prime \prime}+v+\epsilon q_{1}(t) v=0,
$$

and is in fact the method employed by McLachlan [1].*

The idea here, however, is to work directly with (3). It was found in dealing with the stability of subharmonics of the forced van der Pol equation [2] that the transformation (4) and the resulting Hill equation (5) were cumbersome to work with when the desired information was only whether $u(t)$ was a function which increased, decreased, or remained periodic with time. (This, of course, is what is meant here by stability. If the small disturbance $u(t)$ grows with time, the original physical system is said to be unstable).

The following approach will be adopted: An analysis due originally to Poincare but used in the form given by Friedrichs [3] will be applied to (3) to discover if periodic analytic solutions to (3) exist. From the general Floquet theory [4] and the theorems of Haupt [5] for the Hill equation we are led to expect that these periodic solutions will form the boundaries between the stable and unstable regions. Once assured that there are periodic analytic solutions (analyticity in $\epsilon$ is implied by the general existence theorems; it is only the periodicity of these analytic solutions that is tested), the solution $u(t)$ is expanded in a power series in $\epsilon$. This last named step will again produce a purely periodic solution but will also produce the conditions on $p(t), q_{1}(t)$ and $\omega$ for which stable solutions exist. The feature of this analysis which may be novel is that it is shown that the periodic coefficients need not have exactly the linearized period of (3) but may be somewhat different [according to the form of $p(t)$ and $q_{1}(t)$ ] and still produce a stable solution.

It should be emphasized here that this is intended to provide a quantitative study of the special equation (3) dealt with, and even that only in the restricted region of $\epsilon$ small. Qualitative investigations begin with Liapounoff [6] and have been taken up by other authors.

In order to use Friedrich's approach directly, let $\varphi=\omega t$ and consider a phase shift $\delta$ in $\varphi$ such that the equation (3) now written in the form

$$
u^{\prime \prime}+u=-\epsilon\left[p(\theta+\delta) u^{\prime}+q_{1}(\theta+\delta) u\right]
$$

has the special initial conditions

$$
u(0)=A, \quad u^{\prime}(0)=0 .
$$

Following Friedrichs we introduce the variable $\eta$ such that the period of the resultant motion, $T$, equals $2 \pi+\epsilon \eta(\epsilon)$. Friedrichs then seeks values of $A, \delta$, and $\eta$, taken to be analytic in $\epsilon$, such that periodicity conditions on the solutions near $\epsilon=0$ are satisfied. The method is given fully in the textbook of Stoker [3, p. 233] and need not be repeated here. In the case of a linear equation, $A$ is independent of $\omega t$ and is not involved in the periodicity considerations. The condition for periodicity of solutions finally obtained is:

$$
\eta_{0}=-\pi\left\{c_{0} \mp\left[\frac{1}{4}\left(a_{2}-d_{2}\right)^{2}+\frac{1}{4}\left(c_{2}+b_{2}\right)^{2}-a_{0}^{2}\right]^{1 / 2}\right\} .
$$

\footnotetext{
${ }^{*}$ Numbers in brackets refer to references given at the end of the paper.
} 
Here $\eta_{0}$ is the first term in an expansion of $\eta(\epsilon)$ in powers of $\epsilon$, and the $c_{0}, a_{0}, a_{2}$, $b_{2}, c_{2}, d_{2}$ are the coefficients in the Fourier series expansion of $p$ and $q_{1}$, namely

$$
\begin{aligned}
& p(t)=a_{0}+\sum_{n=1}^{\infty}\left(a_{n} \sin n \omega t+b_{n} \cos n \omega t\right), \\
& q_{1}(t)=c_{0}+\sum_{n=1}^{\infty}\left(c_{n} \sin n \omega t+d_{n} \cos n \omega t\right) .
\end{aligned}
$$

Translating back into terms of $\omega$, we have

$$
\omega=1-\frac{\epsilon \eta_{0}}{2 \pi}=1-\frac{\epsilon}{2}\left\{c_{0} \mp\left[\frac{1}{4}\left(a_{2}-d_{2}\right)^{2}+\frac{1}{4}\left(c_{2}+b_{2}\right)^{2}-a_{0}^{2}\right]^{1 / 2}\right\} .
$$

Thus periodic solutions will exist when the period of the coefficients is given by

$$
T=\frac{2 \pi}{\omega}=2 \pi\left(1+\frac{\epsilon}{2}\left\{c_{0} \mp\left[\frac{1}{4}\left(a_{2}-d_{2}\right)^{2}+\frac{1}{4}\left(c_{2}+b_{2}\right)^{2}-a_{0}^{2}\right]^{1 / 2}\right\}\right) .
$$

Let us now investigate (3) by the customary formal expansion in a power series in $\epsilon$. With the change of variable $\tau=\omega t$, we have

$$
u^{\prime \prime}(\tau)+\frac{\epsilon}{\omega} p(\tau) u^{\prime}(\tau)+\frac{\epsilon}{\omega^{2}}\left(q_{1}(\tau)+\frac{1}{\epsilon}\right) u(\tau)=0 .
$$

It is well known [4] that the fundamental solution to the Floquet equation is given by

$$
u=e^{\lambda \tau} g(\tau),
$$

where $\lambda$ is a complex number and $g(\tau)$ has period $2 \pi$. Let us take $\lambda, g$, and $\omega$ to be analytic functions of the small parameter $\epsilon$ so that we may write the expansions

$$
\begin{aligned}
\lambda & =\lambda_{0}+\epsilon \lambda_{1}+\epsilon^{2} \lambda_{2}+\cdots, \\
g & =g_{0}+\epsilon g_{1}+\epsilon^{2} g_{2}+\cdots, \\
\omega & =1+\epsilon \omega_{1}+\epsilon^{2} \omega_{2}+\cdots,
\end{aligned}
$$

Substituting these into (6), equations of any desired degree in $\epsilon$ are obtained. For the degree zero:

$$
g_{0}^{\prime \prime}+2 \lambda_{0} g_{0}^{\prime}+\left(\lambda_{0}^{2}+1\right)=0 .
$$

Now if the condition that $g$ be periodic is imposed, the $g_{n}$ must be periodic; in particular, $g_{0}$ must have the linearized period, $2 \pi$. This will obtain if

$$
\lambda_{0}= \pm i, \quad g_{0}=c+e^{-2 \lambda_{0} \tau} .
$$

Here one of the integration constants has been taken to be unity with no loss in generality. Proceeding, the equation of first degree in $\epsilon$ can now be found making use of the zeroth order solutions, $\lambda_{0}$ and $g_{0}$. The result is

$$
g_{1}^{\prime \prime}+2 \lambda_{0} g_{1}^{\prime}=-\left(c+e^{-2 \lambda_{0} \tau}\right)\left[2 \lambda_{1} \lambda_{0}-2 \omega_{1}+q_{1}+\lambda_{0} p\right]+2 \lambda_{0} e^{-\lambda_{0} 2 \tau}\left[2 \lambda_{1}+p\right] .
$$

Since the solution $g_{1}$ is to be periodic, the terms on the right hand side of (7) which will produce solutions that are non-periodic must be eliminated. This represents the imposition of the periodicity condition as a boundary condition. The method, often termed the casting out of secular terms, was given by Poincare and used extensively by 
Duffing and many others. The terms on the right hand side of (7) which give rise to secular solutions are the constant terms and the terms with $e^{-2 \lambda_{0} \tau}$ as multiplier. Again we use an expansion of the periodic functions:

$$
\begin{gathered}
p(\tau)=a_{0}+\sum_{n=1}^{\infty} a_{n} \sin n \tau+b_{n} \cos n \tau, \\
q_{1}(\tau)=c_{0}+\sum_{n=1}^{\infty} c_{n} \sin n \tau+d_{n} \cos n \tau .
\end{gathered}
$$

Collecting constants and coefficients of $e^{-2 \lambda_{0} \tau}$, we have

$$
\begin{gathered}
-c\left[2 \lambda_{1} \lambda_{0}-2 \omega_{1}+c_{0}+\lambda_{0} a_{0}\right]-\frac{c_{2}}{2 i}+\frac{\lambda_{0} a_{0}}{2 i}-\frac{d_{2}}{2}+\frac{\lambda_{0} b_{2}}{2}=0, \\
-\left[-2 \lambda_{0} \lambda_{1}-\lambda_{0} a_{0}-2 \omega_{1}+c_{0}\right]-c\left[-\frac{c_{2}}{2 i}+\frac{d_{2}}{2}-\frac{\lambda_{0} a_{2}}{2 i}+\frac{b_{2} \lambda_{0}}{2}\right]=0 .
\end{gathered}
$$

Eliminating $c$ we obtain

$$
2 \lambda_{1}=-a_{0} \pm\left[\frac{1}{4}\left(c_{2}+b_{2}\right)^{2}+\frac{1}{4}\left(a_{2}-d_{2}\right)^{2}-\left(c_{0}-2 \omega_{1}\right)^{2}\right]^{1 / 2} .
$$

To check the previous result, we set $\lambda_{1}=0$ and obtain

$$
\omega_{1}=\frac{c_{0}}{2} \mp \frac{1}{2}\left[\frac{1}{4}\left(c_{2}+b_{2}\right)^{2}+\frac{1}{4}\left(a_{2}-d_{2}\right)^{2}-a_{0}^{2}\right]^{1 / 2} .
$$

Since $\omega=1+\epsilon \omega_{1}$ in this approximation and $\omega=1-\epsilon \eta_{0} / 2 \pi$ has been used, then $\omega_{1}=$ $-\eta_{0} / 2 \pi$. The result is

$$
\eta_{0}=-\pi\left(c_{0} \pm\left[\frac{1}{4}\left(c_{2}+b_{2}\right)^{2}+\frac{1}{4}\left(a_{2}-d_{2}\right)^{2}-a_{0}^{2}\right]^{1 / 2}\right) .
$$

Returning to (8), it is observed:

1) if

$$
\left(c_{0}-2 \omega_{1}\right)^{2} \geq \frac{1}{4}\left[\left(c_{2}+b_{2}\right)^{2}+\left(a_{2}-d_{2}\right)^{2}\right],
$$

stability is determined entirely by $a_{0}$

a) $a_{0}<0, \lambda_{1}>0$, and the resulting $u(t)$ increases with time

b) $a_{0}>0, \lambda_{1}<0$, and $u(t)$ decreases with time.

2) if

$$
\left(c_{0}-2 \omega_{1}\right)^{2}<\frac{1}{4}\left\{\left(c_{2}+b_{2}\right)^{2}+\left(a_{2}-d_{2}\right)^{2}\right\},
$$

stability is determined by

$$
-\frac{a_{0}}{2}+\frac{1}{2}\left[\frac{1}{4}\left\{\left(c_{2}+b_{2}\right)^{2}+\left(a_{2}-d_{2}\right)^{2}\right\}-\left(c_{0}-2 \omega_{1}\right)^{2}\right]^{1 / 2} .
$$

Let us consider as an example to explicate the above work the equation (2) where 
$y_{0}$ will be taken to be $A \sin t$. (This is exactly the problem solved by McLachlan in [1], p. 190). Then

$$
\begin{aligned}
& p(t)=\left[\left\{\frac{A^{2}}{2}-1\right\}-\frac{A^{2}}{2} \cos 2 t\right], \\
& q_{1}(t)=A^{2} \sin 2 t \\
& a_{0}=\frac{A^{2}}{2}-1 . \\
& a_{2}=c_{0}=d_{2}=0, \quad c_{2}=A^{2} ; \quad b_{2}=-\frac{A^{2}}{2}
\end{aligned}
$$

Notice that for periodicity we would need

$$
\omega_{1}=\frac{1}{2}\left(\frac{A^{4}}{16}-\frac{A^{4}}{4}+A^{2}-1\right)^{1 / 2}=0,
$$

since here $\omega=1$. If $A^{2}=4$, then this condition is satisfied. Further, using (8) since $c_{0}-2 \omega_{1}=0$ and $\frac{1}{4}\left\{\left(c_{2}+b_{2}\right)^{2}+\left(a_{2}-d_{2}\right)^{2}\right\}>0$

$$
\lambda_{1}=-\frac{A^{2}}{8}+\frac{1}{2}
$$

Thus $\lambda_{1}<0$ for $A^{2}>4$ and $>0$ for $A^{2}<4$.

This example as was remarked is given by McLachlan [1] and was presented merely to show the ease in which stability characteristics may be obtained once $\lambda_{1}$ is computed in terms of $a_{0}, a_{2}, b_{2}, c_{2}$, and $d_{2}$.

\section{REFERENCES}

1. N. McLachlan, Ordinary non-linear differential equations, Oxford, 1950.

2. H. G. Cohen, Subharmonic synchronization of the forced Van der Pol equation (To appear in the Proceedings of the Colloquium on non-linear vibrations, Île de Porquerolles, August, 1951.

3. J. J. Stoker, Non-linear vibrations, Interscience, New York, 1950. (see especially Appendix I).

4. E. L. Ince, Ordinary differential equations, Dover, New York.

5. M. J. O. Strutt, Lamésche, Mathieusche und verwandte Funktionen in Physik und Technik, Julius Springer, Berlin, 1932.

6. A. Liapounoff, Problème general de la stabilité du mouvement, Princeton University Press, Princeton, 1949.

\section{ON THE RELATIONSHIP BETWEEN THE MARTIENSSON AND DUFFING METHODS FOR NONLINEAR VIBRATIONS*}

By ROBERT E. ROBERSON (Mechanics Division, Naval Research Laboratory)

The background for a number of one-term approximation methods and their application to forced nonlinear vibrations has recently been discussed by Schwesinger. ${ }^{1}$

${ }^{*}$ Received Aug. 15, 1951. This paper corresponds to part of a dissertation submitted to Washington University in partial fulfillment of the requirements for the degree of Doctor of Philosophy.

${ }^{1} \mathrm{G}$. Schwesinger, On one-term approximations of forced nonharmonic vibrations, J. Appl. Mech. 17, 202-208 (1950). Note that he attributes to Rüdenberg the method that is designated here as Martiensson's method. 